\section{Lower respiratory infections in early life are linked to later asthma}

\author{
Theo J Moraes, ${ }^{1}$ Malcolm R Sears ${ }^{2}$
}

Asthma is the most prevalent chronic respiratory disease worldwide. ${ }^{1}$ While much progress has been made to understand the determinants of asthma, why a specific individual develops asthma is not entirely clear. Within this discussion, it should be noted that asthma is not a homogenous disease; that is to say, there are many (endo)types of asthma. ${ }^{2}$ In children, the most common pattern is a T2 exaggerated immune response with eosinophils, interleukin (IL)-4, IL-5 and IL-13 playing important roles. While clearly asthma is a complex disease, some of the noise in the literature surrounding the determinants of asthma is a result of disease definition. The presence of wheezing or reduced $\mathrm{FEV}_{1}$ may represent a number of endotypes of asthma or indeed non-asthma phenotypes.

With this caveat in mind, it is clear that viral infections and allergic sensitisation are key factors associated with a diagnosis of asthma. $^{3}$ Numerous epidemiological and experimental studies have provided this link. A popular paradigm postulates that recurrent respiratory viral infections at critical time periods of immune and lung development in childhood and infancy coupled with allergic sensitisation are associated with the development of asthma. ${ }^{4}$ What is often questioned is the direction of this association; is a child with an underlying asthma phenotype more likely to experience viral infections and develop allergy? Or is an otherwise healthy child who happens to get viral infections subsequently pushed into an asthma phenotype? Like many 'either-or' questions, the answer is likely 'yes'; that is, either possibility or neither may be operational in different situations. Indeed, given that asthma is so heterogeneous, it is logical that there may be many ways to reach this diagnosis.

In Thorax, van Meel et al provide more evidence to link respiratory tract infections in early life with a diagnosis of asthma and

${ }^{1}$ Department of Pediatrics, The Hospital for Sick Children, Toronto, Canada

${ }^{2}$ Department of Medicine, St. Joseph's Healthcare and McMaster University, Hamilton, Canada

Correspondence to Professor Malcolm R Sears, Firestone Institute for Respiratory Health, St. Joseph's Healthcare and McMaster University, Hamilton, Ontario L8N 4A6, Canada; searsm@mcmaster.ca reduced lung function in later life. ${ }^{5}$ This work is based on a large, well-characterised birth cohort from the Netherlands; the Generation R Study. ${ }^{6}$ This population-based cohort offers results that may be more generalisable to the population at large than many previous cohorts which were often 'high risk' and selected based on a history of parental asthma or allergy. Using questionnaires, the occurrence of viral infections during the first 6 years of life was documented. Children then had spirometry performed at the age of 10 years and current asthma at that age was defined as having a physician diagnosis of asthma ever with symptoms or medication in the last 12 months. The authors found that lower respiratory tract infections before the age of 3 years were strongly and consistently associated with lower lung function $\left(\mathrm{FEV}_{1}\right)$ and with a diagnosis of current asthma at age 10. However, the effect on lung function, while significant, was relatively small as judged by change in $\mathrm{z}$-score. Lower respiratory tract infections between the ages of 3 and 6 years were also associated with a diagnosis of asthma, but there was no significant effect of these infections on lung function.

The authors hypothesise that early life respiratory tract infections might have a direct effect on lung development and the risk of asthma and support this position in part based on the statistical modelling employed, namely cross-lagged modelling to infer the direction of the association between infections and asthma. Thus, respiratory tract infections in early life were associated with subsequent reduced lung function and a diagnosis of asthma, but not vice versa. Regardless, this is not an interventional experimental study and any attribution of causation should be considered with that in mind.

There is increasing evidence of genetic interactions with wheezing associated with rhinovirus infections ${ }^{7}$ and studies have identified specific single nucleotide polymorphisms associated with viral-induced wheeze. ${ }^{8}$ Other studies indicate that a deficient immune response to viral infections may predict persistence of asthma. ${ }^{9}$ These and many other studies indicate the substantial complexity of asthma even when limited to that associated with viral infections.
A significant body of work also links allergen sensitisation to subsequent wheezing. ${ }^{10}$ The 'allergic march' has become almost dogma in the field of asthma with susceptible children progressing through allergy, atopic dermatitis, asthma and allergic rhinitis. ${ }^{11}$ So it is interesting that allergic sensitisation did not factor into the associations seen in the Generation $\mathrm{R}$ cohort. The allergens tested were not included in their report but presumably included house dust mite (as 'inhalant allergic sensitisation' was tested); sensitisation to food allergens was not documented. It is possible an effect of allergic sensitisation may have been found with a broader range of allergens or lower threshold for definition of sensitisation or with earlier testing. In our Canadian Healthy Infant Longitudinal Development (CHILD) Study, food sensitisation was dominant at age 1 year, whereas inhalant sensitisation became more evident at 3 and 5 years; availability of measures of infant lung function and preschool lung function and objective viral data in that large general population-based cohort will provide further objective data to address this question. ${ }^{12} 13$

While there have been a number of cohort studies linking early life infections and wheezing to asthma, it is interesting to compare the Generation $\mathrm{R}$ study with the Tucson Children's Respiratory Study. ${ }^{14}$ In that general population-based cohort, wheezing phenotypes were described based on wheezing prevalence over the first 6 years of life; transient wheezers wheezed in the first 3 years, non-atopic wheezers wheezed early and then wheezing prevalence reduced over time. Those defined as having asthma (who also had more atopic sensitisation) had more persistent wheezing though not as prevalent in the first 3 years. This suggests that early life symptoms may be less strongly associated with atopy, similar to what was reported by van Meel et al. Thus, the type of asthma described in this study may include a non-allergic phenotype which may be associated with multiple viral infections in early life in the absence of allergen sensitisation, even though $32 \%$ of the cohort did demonstrate allergic sensitisation at age 10 years (figure 1 ).

van Meel et al also report that gastroenteritis in early life was associated with asthma (but not lung function changes) at age 10 . This association was not generalised to any infection as it was not seen with urinary tract infections (though the numbers of these infections were not large). The authors 


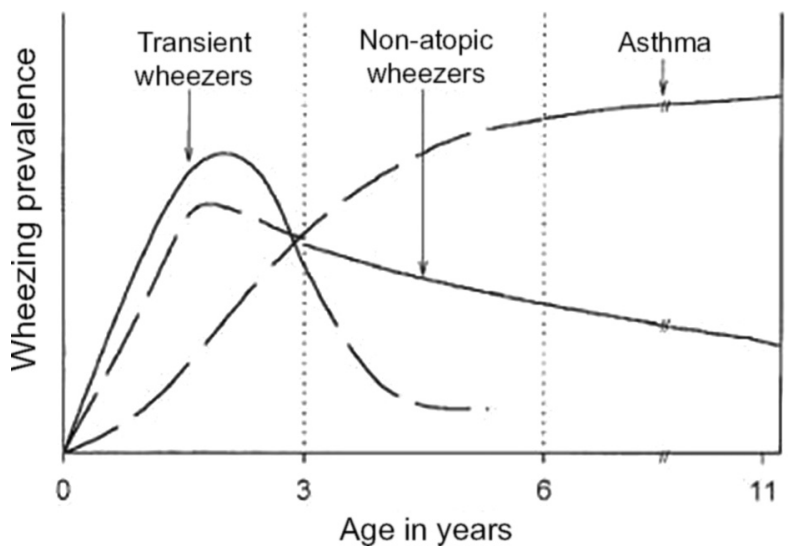

Figure 1 Hypothetical peak prevalence by age for the 3 different wheezing phenotypes. The prevalence for each age interval should be the area under the curve. This does not imply that the groups are exclusive (reproduced with permission from Taussig et $a^{14}{ }^{14}$ ).

suggest that gastrointestinal infections may alter the intestinal microbiome in early life and hence the development of asthma. This is an attractive hypothesis as there is strong experimental evidence to support the role of the microbiome in the first few months of life influencing lung function and asthma. ${ }^{15}$ However, antibiotic use was examined in this study and no associations were noted. Thus, while the association of gastrointestinal infections with asthma is interesting, it remains of uncertain significance.

The authors acknowledge some caveats of the current study, the most important of which are that infections were defined by report and not by microbiological sampling and that asthma was defined by questionnaire and not by objective measures. However, given the size of this study, these limitations are understandable. Further, lung function measures and allergic sensitisation were obtained at a single time point and so longitudinal analysis was not possible. Specifically, it is not clear that lung function was normal prior to viral infections. Finally, while the Generation R cohort is very large, the study population was just over $50 \%$ of the original cohort, and major differences were seen between those included and not included. These differences included factors such as smoking during pregnancy, breastfeeding and day care attendance, all of which are known to influence lung infections and/or lung function. Notwithstanding these limitations, the current study adds to the body of work demonstrating that early life respiratory viral infections are associated with a diagnosis of asthma and reduced lung function.

In summary, while epidemiological studies can never truly determine if viral infections cause or provide the substrate for some variants of asthma, the reality is that this question will not be fully answered until we can define more precisely the multiplicities of phenotypes, genotypes and endotypes of what we know as asthma.

\section{Competing interests None declared.}

Provenance and peer review Commissioned; internally peer reviewed.

(c) Article author(s) (or their employer(s) unless otherwise stated in the text of the article) 2018. All rights reserved. No commercial use is permitted unless otherwise expressly granted.

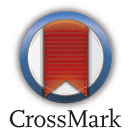

To cite Moraes TJ, Sears MR. Thorax 2018:73:105-106.

Published Online First 23 November 2017

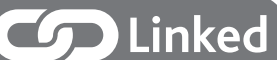

- http://dx.doi.org/10.1136/thoraxjnl-2017-210149

Thorax 2018;73:105-106.

doi:10.1136/thoraxjn|-2017-211104

\section{REFERENCES}

1 GBD 2015 Chronic Respiratory Disease Collaborators. Global, regional, and national deaths, prevalence, disability-adjusted life years, and years lived with disability for chronic obstructive pulmonary disease and asthma, 1990-2015: a systematic analysis for the Global Burden of Disease Study 2015. Lancet Respir Med 2017;5:691-706.

2 Pavord ID, Beasley R, Agusti A, et al. After asthma: redefining airways diseases. Lancet 2017.

3 Burbank AJ, Sood AK, Kesic MJ, et al. Environmental determinants of allergy and asthma in early life. $J$ Allergy Clin Immunol 2017;140:1-12.

4 Holt PG, Sly PD. Viral infections and atopy in asthma pathogenesis: new rationales for asthma prevention and treatment. Nat Med 2012;18:726-35.

5 van Meel ER, den Dekker HT, Elbert NJ, et al. A population-based prospective cohort study examining the influence of early-life respiratory tract infections on school-age lung function and asthma. Thorax 2018;73:167-73.

6 Kooijman MN, Kruithof CJ, van Duijn CM, et al. The generation R study: design and cohort update 2017. Eur J Epidemiol 2016;31:1243-64.

7 Calıškan M, Bochkov YA, Kreiner-Møller E, et al. Rhinovirus wheezing illness and genetic risk of childhood-onset asthma. N Engl J Med 2013;368:1398-407.

8 Amat F, Louha M, Benet M, et al. The IL-4 rs2070874 polymorphism may be associated with the severity of recurrent viral-induced wheeze. Pediatr Pulmonol 2017:52:1435-42.

9 Baraldo S, Contoli M, Bonato $M$, et al. A deficient immune response to viral infections in children predicts later asthma persistence. Am J Respir Crit Care Med Published Online First: 1 Sep 2017. doi: 10.1164/ rccm.201706-1249LE.

10 Gold DR, Adamkiewicz G, Arshad SH, et al. NIAID, NIEHS, NHLBI, and MCAN workshop report: the indoor environment and childhood asthma-implications for home environmental intervention in asthma prevention and management. J Allergy Clin Immunol 2017;140:933-49.

11 Khan SJ, Dharmage SC, Matheson MC, et al. Is the atopic march related to confounding by genetics and early-life environment? a systematic review of sibship and twin data. Allergy 2018;73:17-28.

12 Subbarao P, Anand SS, Becker AB, et al. The Canadian Healthy Infant Longitudinal Development (CHILD) Study: examining developmental origins of allergy and asthma. Thorax 2015;70:998-1000.

13 Moraes TJ, Lefebvre DL, Chooniedass R, et al. The Canadian Healthy Infant Longitudinal Development birth cohort study: biological samples and biobanking. Paediatr Perinat Epidemiol 2015;29:84-92.

14 Taussig LM, Wright AL, Holberg CJ, et al. Tucson children's respiratory study: 1980 to present. J Allergy Clin Immunol 2003;111:661-75.

15 Arrieta MC, Stiemsma LT, Dimitriu PA, et al.Early infancy microbial and metabolic alterations affect risk of childhood asthma. Sci Trans/ Med 2015;7:307ra152. 